\title{
Oromucosal Suspension Dosage Form
}

National Cancer Institute

\section{Source}

National Cancer Institute. Oromucosal Suspension Dosage Form. NCI Thesaurus. Code C149744.

Liquid sing le-dose or multidose preparation consisting of a suspension intended for oromucosal use. 